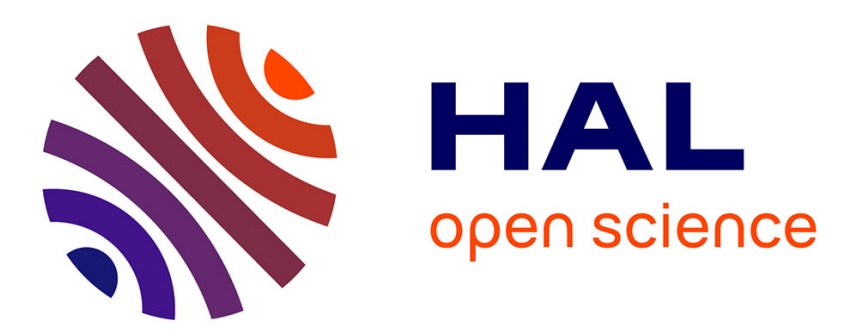

\title{
Optical event horizons from the collision of a soliton and its own dispersive wave
}

S. F Wang, Arnaud Mussot, Matteo Conforti, A. Bendahmane, X. L Zeng, Alexandre Kudlinski

\section{- To cite this version:}

S. F Wang, Arnaud Mussot, Matteo Conforti, A. Bendahmane, X. L Zeng, et al.. Optical event horizons from the collision of a soliton and its own dispersive wave. Physical Review A : Atomic, molecular, and optical physics [1990-2015], 2015, 92 (2), 10.1103/PhysRevA.92.023837 . hal-02388991

\section{HAL Id: hal-02388991 \\ https://hal.science/hal-02388991}

Submitted on 2 Dec 2019

HAL is a multi-disciplinary open access archive for the deposit and dissemination of scientific research documents, whether they are published or not. The documents may come from teaching and research institutions in France or abroad, or from public or private research centers.
L'archive ouverte pluridisciplinaire HAL, est destinée au dépôt et à la diffusion de documents scientifiques de niveau recherche, publiés ou non, émanant des établissements d'enseignement et de recherche français ou étrangers, des laboratoires publics ou privés. 


\title{
Optical event horizons from the collision of a soliton and its own dispersive wave
}

\author{
S. F. Wang, ${ }^{1,2}$ A. Mussot, ${ }^{1}$ M. Conforti, ${ }^{1}$ A. Bendahmane, ${ }^{1}$ X. L. Zeng, ${ }^{2}$ and A. Kudlinski ${ }^{1}{ }^{1, *}$ \\ ${ }^{1}$ Laboratoire PhLAM, UMR CNRS 8523, IRCICA, USR CNRS 3380, Université Lille 1, 59655 Villeneuve d'Ascq, France \\ ${ }^{2}$ Key Laboratory of Specialty Fiber Optics and Optical Access Network, Shanghai University, 200072 Shanghai, China
}

(Received 22 May 2015; published 20 August 2015)

\begin{abstract}
We observe experimentally the spectral signature of the collision between a soliton and the dispersive wave initially emitted from the soliton itself. This collision, interpreted in terms of an optical event horizon, is controlled by the use of an axially varying fiber which allows us to shape both the soliton and dispersive wave trajectories so that they both collide at a precise location within the fiber. The interaction of the dispersive wave with the soliton generates a reflected wave with a conversion efficiency which can be controlled by the input pump power. These experimental results are confirmed by numerical solution of the generalized nonlinear Schrödinger equation and by the analytical calculation of the conversion efficiency.
\end{abstract}

DOI: 10.1103/PhysRevA.92.023837

PACS number(s): 42.65.Tg

\section{INTRODUCTION}

Event horizons can be mimicked in optical fibers by the nonlinear interaction of a weak linear radiation (usually termed the probe wave) with an intense copropagating soliton [1-3]. So-called fiber event horizons (FEHs) occur when the probe wave, traveling at a different group velocity with respect to the soliton, is unable to pass through it during their collision. The probe wave is therefore reflected onto the soliton, which acts as a nonlinear barrier, altering its group velocity. In the spectral domain, this results in the frequency conversion of the probe wave at $\omega_{P}$ into a reflected wave (RW) at $\omega=\omega_{R}$ that satisfies the phase-matching (PM) condition [4-6]

$$
D(\omega)=D\left(\omega_{P}\right),
$$

where $D(\omega)=\beta(\omega)-\beta\left(\omega_{S}\right)-\beta_{1} \times\left(\omega-\omega_{S}\right)$ denotes the wave number in a reference frame moving with the soliton, $\beta(\omega)$ is the fiber propagation constant and $\beta_{1}=\partial_{\omega} \beta\left(\omega_{S}\right)$ is the group velocity at the soliton frequency $\omega_{S}$. The analogy between event horizons and the nonlinear reflection of a weak probe onto a soliton has attracted much interest over the past few years and opens innovative perspectives in the control of light [7-10], in quantum physics [11,12], as well as in superfluidity [13]. The few nonlinear optics experiments on the subject were performed by causing the collision of an intense soliton with a weak probe carefully adjusted at the fiber input in terms of power, wavelength detuning, and delay $[1,3,14-16]$. However, it is still unclear whether this analogy with event horizons persists when the probe parameters are not specifically controlled, as was the case in previous experiments.

Here, we show experimentally that this process is actually very robust, as FEHs can be observed from the collision between a soliton and the dispersive wave (DW) emitted directly from this soliton at the early stage of propagation. The soliton is excited by an ultrashort pulse and generates a phase-matched DW acting as the probe wave. The collision between the soliton and this DW is controlled by using a photonic crystal fiber (PCF) with a longitudinally varying dispersion landscape. The varying dispersion along the fiber

*Corresponding author: alexandre.kudlinski@univ-lille1.fr enhances the deceleration of the soliton [17], reshapes the DW trajectory in the time domain, and also modifies the PM condition [Eq. (1)] so that the DW collides with the soliton in the vicinity of its group velocity matching (GVM) wavelength. This allows us to choose the fiber length at which the FEH is observed, so that the entire process occurs within a few meters.

The paper is organized as follows. In Sec. II we review the physics of a fiber event horizon in the framework of the theory of soliton-DW mixing that gives an analytical estimation of the conversion efficiency. In Sec. III we describe the principle of the experiment, whose results are reported in Sec. IV and discussed in Sec. V. We draw our conclusions in Sec. VI.

\section{FIBER EVENT HORIZONS AND SOLITON-DISPERSIVE WAVE INTERACTION THEORY}

FEHs can be rigorously described by means of the theory of the mixing between a soliton and a DW [5]. We consider the nonlinear Schrödinger equation (NLSE) with third-order dispersion (TOD):

$$
i \partial_{z} u-\frac{\beta_{2}}{2} \partial_{t}^{2} u-i \frac{\beta_{3}}{6} \partial_{t}^{3} u+\gamma|u|^{2} u=0 .
$$

Raman effect and dispersion terms with an order higher than 3 are neglected in order to keep the analysis as simple as possible and to consider only the essential physical effects. Indeed, it has been shown that the Raman deceleration does not play a fundamental role in the process [3].

Consider the solution as the sum of a fundamental soliton $u_{s}$ and linearly dispersing waves $g$ [5]:

$$
u(z, t)=u_{s}(t) e^{i k_{s} z}+g(z, t),
$$

where $u_{s}(t)=\sqrt{P} \operatorname{sech}\left(t / T_{0}\right), \quad P=\left|\beta_{2}\right| /\left(\gamma T_{0}^{2}\right)$, and $k_{s}=$ $\gamma P / 2$. By inserting Eq. (3) in NLSE Eq. (2) and linearizing, at first order we obtain an evolution equation for the perturbation

$$
\begin{aligned}
& i \partial_{z} g-\frac{\beta_{2}}{2} \partial_{t}^{2} g-i \frac{\beta_{3}}{6} \partial_{t}^{3} g+ \\
& \quad-i \frac{\beta_{3}}{6} \partial_{t}^{3}\left|u_{s}\right| e^{i k_{s} z}+\gamma\left|u_{s}\right|^{2} g^{*} e^{2 i k_{s} z}+2 \gamma\left|u_{s}\right|^{2} g=0 .
\end{aligned}
$$

Consider now that DWs are the sum of the resonant radiation emitted from the soliton and other linear waves (i.e., the probe) 


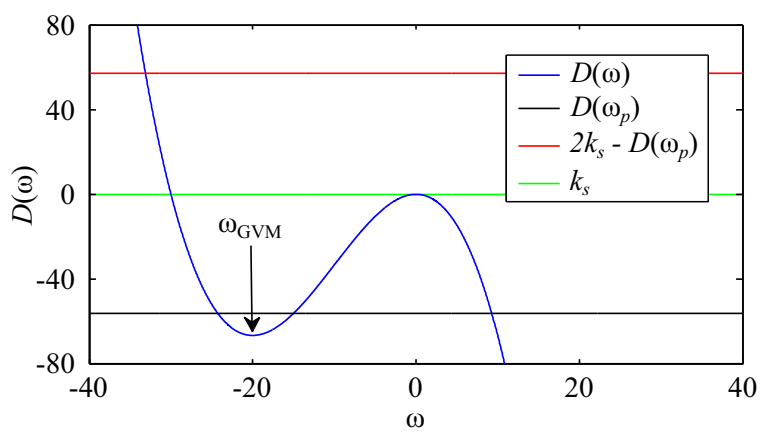

FIG. 1. (Color online) Graphical solution of Eqs. (6)-(8) for a toy model with the following values of (nondimensional) parameters: $\beta_{2}=1, \beta_{3}=-0.1, \gamma=1$.

generated elsewhere:

$$
g(z, t)=w e^{i D\left(\omega_{p}\right) z-i \omega_{p} t}+\psi(z, t),
$$

where $D(\omega)$ is the linear dispersion relation that, when considering TOD only, reads $D(\omega)=\beta_{2} \omega^{2} / 2+\beta_{3} \omega^{3} / 6$. We can recognize three source terms in the second line of Eq. (4) that can force the system efficiently if the following resonance conditions are fulfilled [5]:

$$
\begin{gathered}
D(\omega)=k_{s}, \\
D(\omega)=2 k_{s}-D\left(\omega_{p}\right), \\
D(\omega)=D\left(\omega_{p}\right) .
\end{gathered}
$$

Equation (6) describes the condition for generating the standard resonant radiation (RR) [18], whereas Eqs. (7) and (8) describes two additional peaks generated by four-wave mixing between the soliton and the probe wave. It is easy to see that only Eq. (8) can describe interactions near the GVM point $\omega_{G V M}$ (see Fig. 1). If we consider a probe whose frequency is near GVM frequency, we can study the behavior of the generated radiation by investigating the following reduced equation, where only the source term that is almost in phase (resonant) with the radiation has been considered:

$$
i \partial_{z} \psi-\frac{\beta_{2}}{2} \partial_{t}^{2} \psi-i \frac{\beta_{3}}{6} \partial_{t}^{3} \psi+2 \gamma\left|u_{s}(t)\right|^{2} \psi=0 .
$$

At the GVM point, second-order dispersion changes sign $\left(\beta_{2}=\beta_{2}\left(\omega_{s}\right)=-\beta_{2}\left(\omega_{G V M}\right)\right)$ and TOD can be neglected at first order. By a phase rotation, we can rewrite Eq. (9) taking the GVM frequency as the carrier:

$$
i \partial_{z} \tilde{\psi}+\frac{\beta_{2}}{2} \partial_{t}^{2} \tilde{\psi}+2 \gamma\left|u_{s}(t)\right|^{2} \tilde{\psi}=0
$$

By fixing $\tilde{\psi}=a(t) \exp [i k z]$ we obtain eventually a timeindependent Schrödinger equation ( $z$ independent in fiberoptic notation) in the form

$$
\left(-\frac{\left|\beta_{2}\right|}{2} \frac{d^{2}}{d t^{2}}+V(t)-k\right) a(t)=0,
$$

where the potential induced by the soliton is

$$
V(t)=2 \frac{\left|\beta_{2}\right|}{T_{0}^{2}} \operatorname{sech}^{2}\left(\frac{t}{T_{0}}\right),
$$

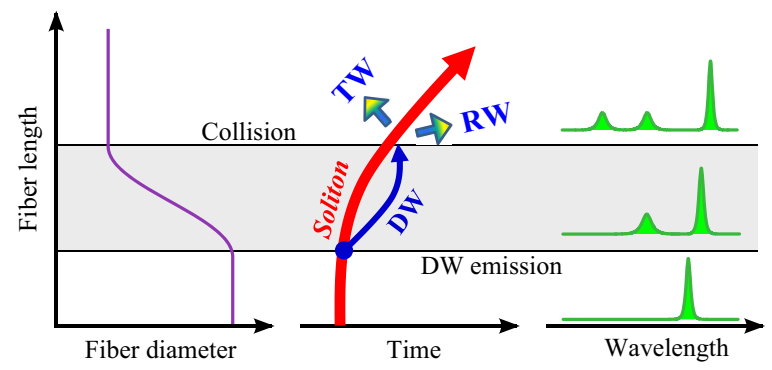

FIG. 2. (Color online) Schematic illustration of the FEH experiment using an axially varying fiber. The gray region corresponds to the shaping of the DW trajectory induced by the axial variation of fiber dispersion. TW, transmitted wave through the soliton (same wavelength as DW); RW, reflected wave on the soliton.

and the wave number of the linear waves is fixed by the approximated dispersion relation $(\mathrm{TOD}=0)$ as $k=\left|\beta_{2}\right| \Omega^{2} / 2$ ( $\Omega$ is the frequency shift from $\omega_{G V M}$ ).

The reflection and transmission coefficients can be calculated analytically for this potential as [19]

$$
\begin{aligned}
& \rho(\Omega)=\frac{\cosh ^{2}\left(\frac{\sqrt{15}}{2} \pi\right)}{\sinh ^{2}\left(\pi \Omega T_{0}\right)+\cosh ^{2}\left(\frac{\sqrt{15}}{2} \pi\right)}, \\
& \tau(\Omega)=\frac{\sinh ^{2}\left(\pi \Omega T_{0}\right)}{\sinh ^{2}\left(\pi \Omega T_{0}\right)+\cosh ^{2}\left(\frac{\sqrt{15}}{2} \pi\right)} .
\end{aligned}
$$

Interestingly, with our approximations, the conversion efficiency $\rho$ does not depend on $\beta_{2}$ for a fundamental soliton. Equations (13) and (14) have been obtained before in Refs. $[3,16]$ by resorting to the concept of comoving-frequency conservation. Our calculations show that we can rigorously describe FEHs in the frame of NLSE, without evoking any relativistic argument.

Equations (13) and (14) indicate that a perfect horizon with $100 \%$ reflection is only realized with an incident wave precisely at the GVM frequency $(\Omega=0)$. However, in this case the probe wave and soliton propagate at exactly the same velocity, and they would require infinite distance to interact. This suggests that an event horizon in a strict sense is unlikely to be observed in optical fibers where a fundamental soliton interacts with a linear wave [20]. Total reflection could in principle be obtained by the scattering with other nonlinear pumps (for instance a higher-order soliton or a shock wave), as shown numerically in Refs. [10,12].

\section{DESIGN OF THE EXPERIMENT}

The schematic diagram shown in Fig. 2 illustrates the principle of our experiment. The fundamental soliton excited by the pump pulse initially emits a DW at shorter wavelength, traveling ahead of the soliton in the time domain (middle panel) [21]. The PM relation describing this process [18,21] gives the frequency of the DW, $\omega_{D W}$, as the root of Eq. (6). Subsequent to the DW emission, the soliton undergoing Raman-induced selffrequency shift (RISFS) decelerates along the fiber, while the DW experiences a sudden temporal deflection (within the gray area in Fig. 2), owing to the axial dispersion variation (see left panel). Simultaneously, this dispersion variation enhances the 


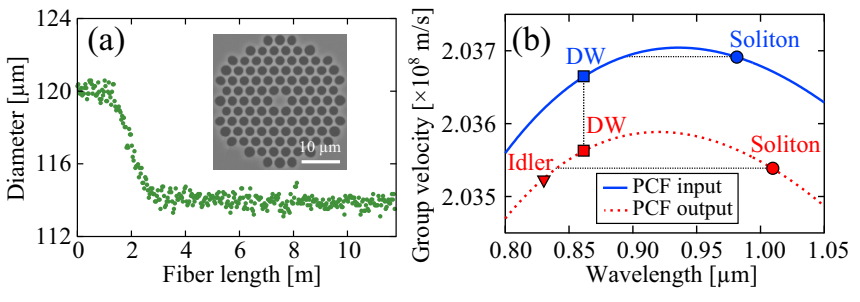

FIG. 3. (Color online) (a) Longitudinal fiber diameter profile measured during the drawing process. Inset: SEM image of the PCF input cross section. (b) Calculated group velocity at the input (top solid blue line) and output (bottom dotted red line) of the PCF. Markers correspond to various waves involved in the experiment of Fig. 4(a) and horizontal lines depict GVM wavelengths with the soliton.

soliton deceleration [17], resulting in their inevitable collision. A part of the DW is transmitted through the soliton, and the remaining part of the DW is blocked and reflected onto it due to the strong nonlinear phase modulation from the soliton. In the spectral domain (right panel), the RW corresponds to the generation of another spectral component following the PM relation (6), which is the signature of the FEH. Note that in principle the spectral signature of the collision between a soliton and its own DW should be observable experimentally in uniform fibers as it is likely to occur in the early stages of most supercontinuum generation experiments [6]. However, it has never been clearly observed to date in this context as it is usually hidden in the supercontinuum spectrum [22,23], due to many other nonlinear effects occurring at the same time, including DW trapping by the soliton [6]. To our knowledge, its experimental observation has thus never been done unambiguously in any optical fiber, tapered or not.

\section{RESULTS}

The longitudinal profile of the PCF diameter used in experiments is shown in Fig. 3(a). It has a uniform initial 1.3$\mathrm{m}$-long section, followed by a 2 -m-long sine shape transition over which the diameter (and therefore the overall structure) is reduced by $5 \%$. The final section is $8.5 \mathrm{~m}$ long and is uniform until the fiber output $(11.8 \mathrm{~m})$. Dispersion properties were calculated from scanning electron microscope (SEM) images [see the input in the inset of Fig. 3(a)]. Figure 3(b) shows the calculated group index curves at the PCF input (top solid blue line) and output (bottom dashed red line). Markers depict the locations of the soliton, DW, and RW involved in the process, as described below. The input and output zero dispersion wavelengths (ZDWs) are 933 and $918 \mathrm{~nm}$ respectively, and the corresponding nonlinear parameters are 27 and $30 \mathrm{~W}^{-1} \mathrm{~km}^{-1}$. Experiments are performed using Fourier transform-limited Gaussian pulses from a Ti:Sa oscillator at $80 \mathrm{MHz}$. They have a full width at half-maximum duration of $130 \mathrm{fs}$ and they are centered at $963 \mathrm{~nm}$. They are directly launched into the PCF after passing a pair of half-wave plates and a polarizer for adjusting the polarization state and input power. The spectral dynamics is recorded by cutting back the fiber every $0.5 \mathrm{~m}$ and successively measuring the output spectrum.

Figure 4(a) displays the experimental spectral dynamics for a pump peak power of $90 \mathrm{~W}$. Corresponding numerical
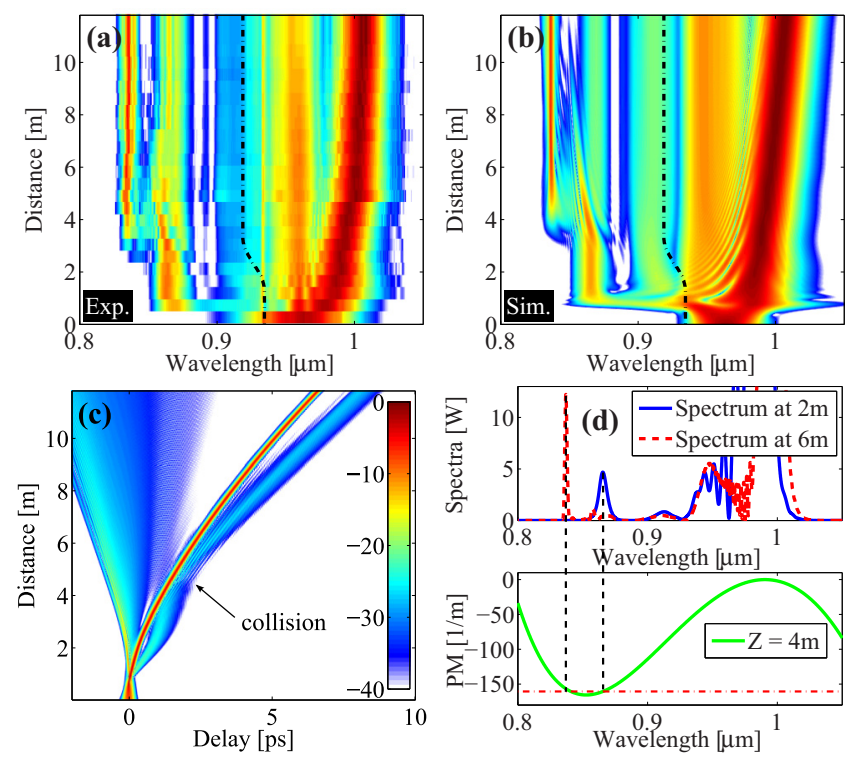

FIG. 4. (Color online) Measured (a) and simulated (b) spectral dynamics illustrating a FEH with a pump peak power of $90 \mathrm{~W}$. Black dashed lines depict the ZDW. (c) Simulated temporal dynamics. (d) Top: simulated spectra at fiber lengths of $2 \mathrm{~m}$ (solid blue line) and $6 \mathrm{~m}$ (dashed red line), i.e., respectively before and after collision. Bottom: graphical solution of PM equation (6) at $4 \mathrm{~m}$. Green solid curve, $D(\omega)$; horizontal dashed red line, $D\left(\omega_{P}\right)$.

simulations are shown in Figs. 4(b) and 4(c) (respectively in the spectral and time domain). They were performed using a generalized nonlinear Schrödinger equation taking the full frequency dependance of the dispersion curve into account, as well as Kerr (including self-steepening) and Raman nonlinearities, with the parameters extracted from the experiment. The spectral dynamics is in excellent quantitative agreement with the cutback measurement of Fig. 4(a). The dynamics scenario is as follows. The pump pulse excites a fundamental soliton, which first emits a DW at $865 \mathrm{~nm}$ according to Eq. (6) within the first meter, due to its proximity to the input ZDW. The soliton then undergoes RISFS, which results in its deceleration in the time domain [Fig. 4(c)]. Then, the variation of the group velocity along the transition region makes the soliton further decelerate and simultaneously reshapes the DW trajectory in the time domain. This induces an inevitable collision between the soliton and the DW around $4 \mathrm{~m}$ [Fig. 4(c)]. In the spectral domain, this results in the conversion of the DW into a RW centered at $837 \mathrm{~nm}$ according to the PM process described by Eq. (1). In our experiment, nearly $85 \%$ of the DW energy before collision [solid blue line in Fig. 4(d)] is converted into the RW after collision [dashed red line in Fig. 4(d)]. A remarkable reflection of the DW on the soliton is also apparent in the temporal domain, which is the signature of a FEH [1,20]. The bottom curve of Fig. 4(d) confirms that the generated RW follows the PM relation of Eq. (1) involving the colliding DW and soliton. Note that in our experimental configuration, the peak power of the DW is typically about $25 \mathrm{~dB}$ lower than the one of the soliton, which is the reason why the modifications of the soliton properties highlighted in Refs. $[8,24]$ are not significant enough to be observable in our case. 

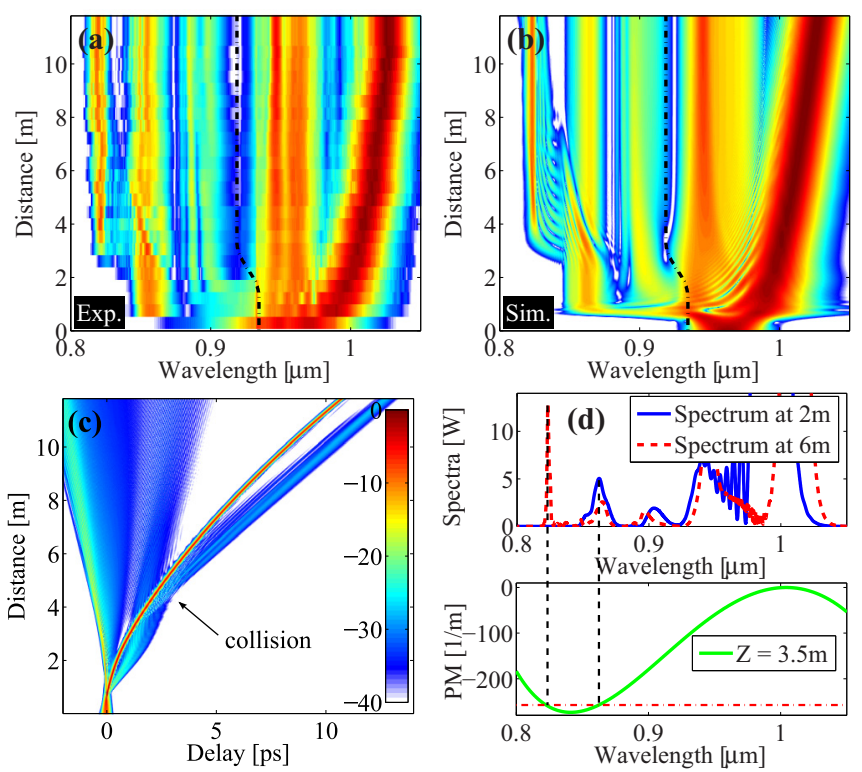

FIG. 5. (Color online) Same as Fig. 4 but for a higher pump peak power of $120 \mathrm{~W}$.

We also performed an alternative experiment during the cutback measurement, corresponding to a slightly higher peak power of $120 \mathrm{~W}$. The results are illustrated in Fig. 5. The scenario is comparable to the first case shown in Fig. 4. However, because the rate of the RISFS is increased, its collision with the DW occurs at a slightly shorter distance, so that the parameters characterizing the process are slightly modified. As a consequence, the PM relation is also modified, leading to a blue shift of the RW and to a change of the conversion efficiency as compared to the previous case. This will be further discussed in Sec. V.

In order to further illustrate the process and gain more insight, we studied numerical spectrograms to elucidate the pulse dynamics simultaneously in the temporal and spectral domains. We use the first case corresponding to Fig. 4 as an example. Figures 6(a)-6(d) show four spectrograms at fiber lengths of 3,6 , and $9 \mathrm{~m}$ and at the output, respectively. The black dashed line represents the ZDW, while the blue and red ones correspond to PM relation of Eqs. (1) and (6), respectively. At the beginning [Fig. 6(a)], the emission of the DW from the soliton follows Eq. (6). They are well separated in the time domain because they travel at different group velocities, as illustrated by blue (gray, top) markers in Fig. 3(b), which are not on the same horizontal line. In the subsequent propagation, the transition region of the fiber allows the soliton-DW collision to take place in the time domain [Fig. 6(b)], which results in the generation of the RW at the short-wavelength edge, in good agreement with Eq. (1) (bottom dashed line). Upon further evolution [Figs. 6(c) and 6(d)], the soliton continuously shifts to longer wavelengths due to RISFS, while the DW and RW evolve toward opposite directions with respect to the soliton in the time domain. This is due to the fact that the RW has a lower group velocity than the soliton, while the DW group velocity is larger than the soliton one, as shown by red (gray, bottom) markers in Fig. 3(b).
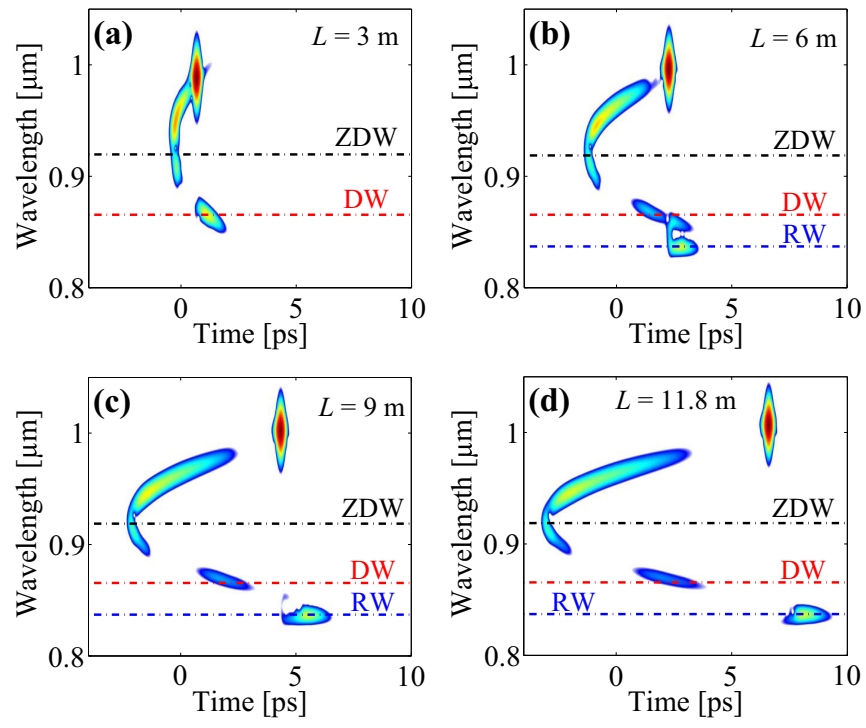

FIG. 6. (Color online) Simulated spectrograms at propagation distances (a) of $3 \mathrm{~m}$, (b) of $6 \mathrm{~m}$, (c) of $9 \mathrm{~m}$, and (d) at the output (see Supplemental Material [25]). Black dashed lines depict the ZDW. Red (gray, middle) and blue (gray, bottom) dashed lines are the calculated PM wavelengths of the DW and RW, respectively, from Eqs. (6) and (1).

\section{DISCUSSION}

We now direct our attention to clarify the different conversion efficiencies into the RW from Figs. 4 and 5, which was pointed out above. Previous studies of FEHs using pump-probe configurations pointed out the role of the probe wavelength in the conversion efficiency into the idler wave [20]. In our case, the Raman soliton acts as the pump wave and the DW initially emitted from the soliton acts as the probe wave. Therefore, we first extracted all parameters of these two waves from numerical simulations of Figs. 4 and 5, before the collision occurs (at fiber lengths of 3.3 and $3.0 \mathrm{~m}$ respectively). Then, we kept them constant except for the probe wavelength, which has been artificially tuned (which is equivalent to vary its group velocity relatively to the soliton) and studied the conversion efficiency into the RW after collision with the soliton. Dashed blue and dash-dot red lines in Fig. 7 show the conversion efficiency versus probe wavelength obtained for the cases of Figs. 4 (90 W input peak power) and 5 (120 W input peak power), respectively. Markers of green square and red rotundity are experimental conversion efficiencies extracted from measurements of Figs. 4(a) and 5(a) respectively. The soliton duration $T_{0}$ has been calculated from the experimental spectra, assuming a transform-limited hyperbolic secant pulse. The dotted green curve and triangle marker correspond to a third experiment (not detailed here) performed in the same conditions as above but with a lower input peak power of $55 \mathrm{~W}$. Concerning the dashed blue line (case of Fig. 4), we extracted the pulse parameters at $3.3 \mathrm{~m}$, where the soliton wavelength is around $990 \mathrm{~nm}$, corresponding to a GVM wavelength of $851 \mathrm{~nm}$. As a consequence, the conversion efficiency tends to unity as the probe wavelength approaches the GVM wavelength [20]. In our experimental conditions, we measured a conversion efficiency of $85 \%$ (blue square marker) in this case. 


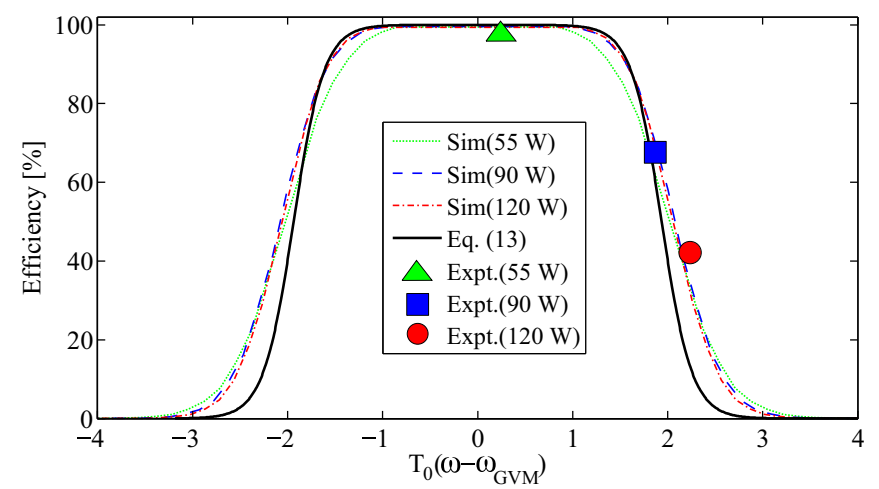

FIG. 7. (Color online) Simulated conversion efficiencies into the idler wave vs probe wavelength, for pump powers of 55, 90, and $120 \mathrm{~W}$ (dotted green, dashed blue, and dash-dot red lines respectively). The corresponding experimental results are shown as the markers of green triangle, blue square, and red rotundity, assuming soliton durations of 95, 68, and 53 fs. Thick black line is the analytically calculated conversion efficiency from Eq. (13).

Note that a $100 \%$ conversion efficiency is not reachable in this case, thus preventing the existence of an event horizon in strict sense. This is caused by the fact that soliton and DW located exactly at GVM wavelengths cannot collide [20]. Around this point, the conversion efficiency decreases symmetrically to zero with increasing probe detuning. The same reasoning can be applied to the dash-dot red and solid black curves. For the dash-dot red line corresponding to the case of Fig. 5, the soliton and DW characteristics are different due to a higher input peak power. The RISFS is strengthened, enabling the soliton to reach a longer wavelength so that its GVM wavelength (around which the overall conversion efficiency curve is centered) is shorter. Another consequence of the higher input peak power is that the DW acting as the probe wave (represented by the red rotundity marker in Fig. 7) is located at a shorter wavelength and farther away from the GVM wavelength. Therefore, the conversion efficiency into the RW is reduced to $45 \%$ in this case, and the remaining part of the DW is transmitted through the soliton, as can be seen from Fig. 5(c). The dotted green line corresponds to a lower input peak power, so that the RW is generated much closer to the GVM wavelength, and thus the experimental conversion efficiency reaches $98 \%$ in this case. This demonstrates that the conversion efficiency into the RW can be investigated by simply adjusting the input peak power in our experimental scheme, which is equivalent to tuning the probe wavelength in pump-probe configurations.

In all cases, the simulated and measured conversion efficiencies are in remarkably good agreement with the analytical curve obtained using Eq. (13) derived from the theory presented in Sec. II.

\section{CONLUSION}

In summary, we have experimentally reported for the first time the spectral signature of the collision between a soliton and its own DW in an optical fiber. Although it is not a prerogative of this observation, the use of an axially varying fiber allows control of the collision process so that the spectral signatures of transmitted and reflected waves are unambiguously observed. The conversion efficiency of the process can be easily adjusted from a change of input power. The measured conversion efficiencies are in excellent agreement with realistic numerical simulations and with the analytical formula we obtained from the soliton-DW mixing theory.

\section{ACKNOWLEDGMENTS}

This work was partly supported by the ANR TOPWAVE (ANR-13-JS04-0004) and NoAWE (ANR-14-ACHN-0014) projects, and by the Fonds Européen de Développement Economique Régional, Labex CEMPI (ANR-11-LABX0007), and Equipex FLUX (ANR-11-EQPX-0017) through the Programme Investissements d'Avenir. S.F.W. acknowledges the grant of the China Scholarship Council (Grant No. 201406890058). X.L.Z. acknowledges the research support by the National Natural Science Foundation of China (Grant No. 11274224) and the Program for Professor of Special Appointment (Eastern Scholar) at Shanghai Institutions of Higher Learning.
[1] T. G. Philbin, C. Kuklewicz, S. Robertson, S. Hill, F. König, and U. Leonhardt, Fiber-optical analog of the event horizon, Science 319, 1367 (2008).

[2] D. Faccio, T. Arane, M. Lamperti, and U. Leonhardt, Optical black hole lasers, Class. Quant. Gravity 29, 224009 (2012).

[3] S. Robertson and U. Leonhardt, Frequency shifting at fiberoptical event horizons: The effect of the Raman deceleration, Phys. Rev. A 81, 063835 (2010).

[4] A. V. Yulin, D. V. Skryabin, and P. J. Russell, Four-wave mixing of linear waves and solitons in fibers with higher-order dispersion, Opt. Lett. 29, 2411 (2004).

[5] D. V. Skryabin and A. V. Yulin, Theory of generation of new frequencies by mixing of solitons and dispersive waves in optical fibers, Phys. Rev. E 72, 016619 (2005).

[6] D. V. Skryabin and A. V. Gorbach, Colloquium: Looking at a soliton through the prism of optical supercontinuum, Rev. Mod. Phys. 82, 1287 (2010).
[7] S. Hill, C. E. Kuklewicz, U. Leonhardt, and F. König, Evolution of light trapped by a soliton in a microstructured fiber, Opt. Express 17, 13588 (2009).

[8] A. Demircan, S. Amiranashvili, and G. Steinmeyer, Controlling light by light with an optical event horizon, Phys. Rev. Lett. 106, 163901 (2011).

[9] A. Demircan, S. Amiranashvili, C. Brée, C. Mahnke, F. Mitschke, and G. Steinmeyer, Rogue events in the group velocity horizon, Sci. Rep. 2, 850 (2012).

[10] E. Rubino, A. Lotti, F. Belgiorno, S. L. Cacciatori, A. Couairon, U. Leonhardt, and D. Faccio, Soliton-induced relativistic-scattering and amplification, Sci. Rep. 2, 932 (2012).

[11] F. Belgiorno, S. L. Cacciatori, M. Clerici, V. Gorini, G. Ortenzi, L. Rizzi, E. Rubino, V. G. Sala, and D. Faccio, Hawking radiation from ultrashort laser pulse filaments, Phys. Rev. Lett. 105, 203901 (2010). 
[12] D. Faccio, Laser pulse analogues for gravity and analog Hawking radiation, Contemp. Phys. 53, 97 (2012).

[13] S. Batz and U. Peschel, Superfluid motion of light past a solitonic obstacle, Phys. Rev. A 85, 033824 (2012).

[14] K. E. Webb, M. J. Erkintalo, Y. Xu, N. Broderick, J. M. Dudley, G. Genty, and S. G. Murdoch, Nonlinear optics of fibre event horizons, Nat. Commun. 5, 4969 (2014).

[15] L. Tartara, Frequency shifting of femtosecond pulses by reflection at solitons, IEEE J. Quantum Electron. 48, 1439 (2012).

[16] A. Choudhary and F. König, Efficient frequency shifting of dispersive waves at solitons, Opt. Express 20, 5538 (2012).

[17] J. C. Travers and J. R. Taylor, Soliton trapping of dispersive waves in tapered optical fibers, Opt. Lett. 34, 115 (2009).

[18] N. Akhmediev and M. Karlsson, Cherenkov radiation emitted by solitons in optical fibers, Phys. Rev. A 51, 2602 (1995).

[19] L. D. Landau and E. M. Lifshitz, Quantum Mechanics (Nonrelativistic Theory) (Pergamon, London, 1977), p. 8081.

[20] G. Genty, M. Erkintalo, and J. M. Dudley, Do optical event horizons really exist? The physics of nonlinear reflection at a soliton boundary, in Advanced Photonics Congress, OSA Technical Digest (online) (Optical Society of America, Massachusetts, 2012), p. NW3D.2.

[21] P. K. A. Wai, C. R. Menyuk, Y. C. Lee, and H. H. Chen, Nonlinear pulse propagation in the neighborhood of the zero-dispersion wavelength of monomode optical fibers, Opt. Lett. 11, 464 (1986)

[22] A. V. Gorbach, D. V. Skryabin, J. M. Stone, and J. C. Knight, Four-wave mixing of solitons with radiation and quasinondispersive wave packets at the short-wavelength edge of a supercontinuum, Opt. Express 14, 9854 (2006).

[23] R. Driben, F. Mitschke, and N. Zhavoronkov, Cascaded interactions between Raman induced solitons and dispersive waves in photonic crystal fibers at the advanced stage of supercontinuum generation, Opt. Express 18, 25993 (2010).

[24] L. Tartara, Soliton control by a weak dispersive pulse, J. Opt. Soc. Am. B 32, 395 (2015).

[25] See Supplemental Material at http://link.aps.org/supplemental/ 10.1103/PhysRevA.92.023837 for simulated spectrograms. 